\title{
Construction of an old-map framework for promoting historical GIS research and education
}

\author{
Keiji YANO $^{\text {a, }}$ *, Satoshi IMAMURA ${ }^{\text {b }}$, Ryo KAMATA ${ }^{\mathrm{c}}$, Muneyuki NATSUME ${ }^{\text {a }}$, Benjamin \\ LEWIS $^{\mathrm{d}}$ \\ ${ }^{a}$ Ritsumeikan University,Keiji Yano yano@lt.ritsumei.ac.jp, Muneyuki Natsume,natsume@fc.ritsumei.ac.jp \\ ${ }^{b}$ CAD CENTER CORPORATION, Satoshi Imamura, sat-imamura@cadcenter.co.jp \\ ${ }^{c}$ Geolonia Inc., Ryo Kamata, ryo@geolonia.com \\ dHarvard University, Benjamin Lewis,blewis@cga.harvard.edu \\ * Corresponding author
}

Keywords: Japanese old maps, Historical GIS, Georeference, Portal site, Digital humanities

\begin{abstract}
'Historical GIS', a fusion of historical geography and geographic information science, has undergone rapid development within Japan and overseas since the 2000s as part of the digital humanities (DH), and involving collaboration between research fields in both the humanities and sciences. However, much geospatial informationsuch as drawings, maps, registers, statistics, and other data from Japan's recent past - remains undigitized and so unavailable in GIS form, unshared, and unavailable to the public. In order to expand historical GIS understanding regarding Japan's recent past and modern times, it will be necessary to promote the digitization of geospatial information, much of which is paper-based, convert it in an appropriate manner to GIS, and share it with the public. Also, in order to promote the expanded use of historical GIS among historical researchers who do not specialize in GIS, such as museum curators, and school instructors (among others), it will be necessary to establish a simple framework that facilitates online searches, GIS analysis, etc. together in one place.
\end{abstract}

The goal of this study is to create 1) 'an ARC (Art Research Center, Ritsumeikan University) Map Portal Database' to serve as a portal to Japan's old maps; 2) 'a Japanese version of Map Warper'; and 3) 'Japanese Old Maps Online' all of which are necessary to create this framework. With this framework, it will be possible for users to choose old maps they want to analyse from 'the ARC Map Portal Database' that allows old maps to be searched across multiple possessing institutions, share old map georeferencing via 'the Japanese version of Map Warper' and other maps converted to GIS with other sites; and, finally, import this material to 'Japanese Old Maps Online' to create maps for detailed analysis, on a variety of subjects.

In order to flesh out 1) 'the ARC Map Portal Database', it will be necessary to proceed with the digitization of the old maps owned by institutions in Japan and abroad and make them available on the web. If the knowledge to do so is not present, or if the funding for digitization and a web release is insufficient, then it is possible to make the maps available through requesting cooperation for common use and collaborative research through institutions such as ARC. It will also be necessary to offer old map metadata in multiple languages and standardize old map titles so that they can be searched in at least both Japanese and English.

Also, regarding 2) 'the Japanese version of Map Warper', when possessing institutions release their old maps to the public, it is desirable for them to offer a license for free non-commercial use of the images (for example, a CC BY 4.0 license). There are also plans in the future to introduce a system where, when users discover old maps on the portal in 1) they want to overlay with present maps and display, they can upload the images to the Japanese-language version of Map Warper with the touch of a button. Georeferences on the Japanese version of Map Warper can basically be uploaded by the creator, with anyone able to participate in the work. Voluntary crowdsourced georeferencing should be solicited in order to increase the number of georeferenced old maps.

Finally, as for 3) 'Japanese Old Maps Online', the construction of such a platform using ArcGIS Hub as a base is currently under consideration (Figure 3). Therefore, there is a desire to enable GSI (Geospatial Information Authority of Japan) maps and a variety of current maps that can be used as base maps to be incorporated into the system, as well as to use 'the Japanese version of Map Warper' from 2) like Hhypermap, (which is a global registry of web map services searching large geospatial catalog by time and space by CGA, Harvard) to search, select, and incorporate georeferenced old Japanese maps. The project also seeks to incorporate open vector data such as digital national land information, eStat (a portal site for Japanese Government Statistics), and basic cartographic information.

Through this framework, it is now possible even for GIS novices to work with data or old maps they themselves or other users have georeferenced easily, through the internet - as well as to conduct analysis using a variety of maps and data. Establishing this sort of environment should lead to further advancements in historical GIS research and education. This framework could also be labelled useful for GIS studies in 'Integrated Geography' and 'Integrated History' beginning as high school geography and history subjects in the 2022 school year. 


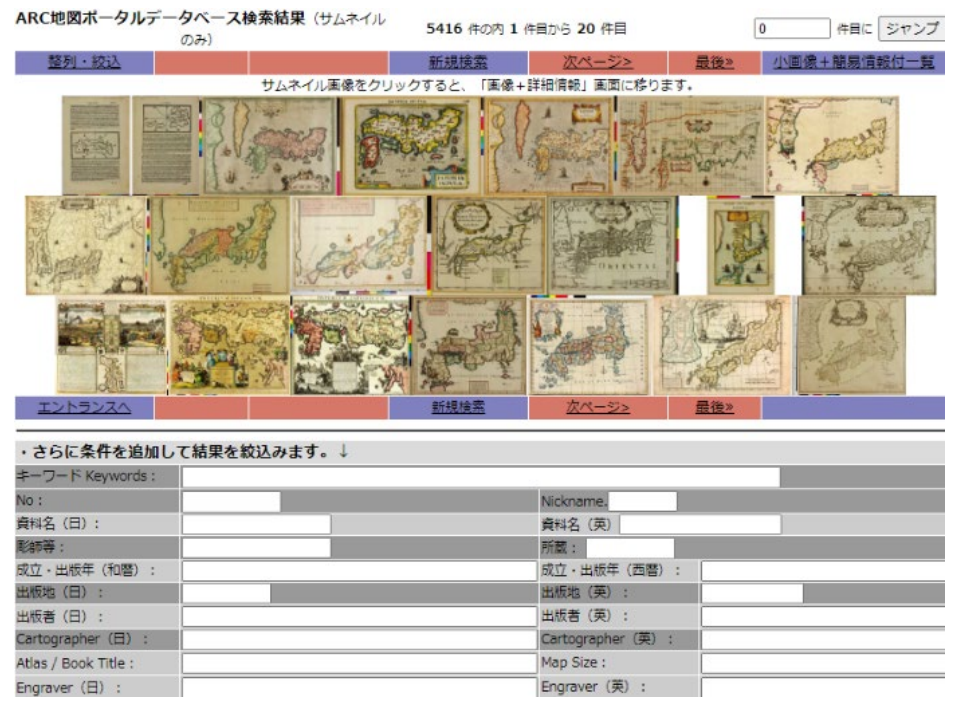

Figure 1. The ARC Map Portal Database.

(https://www.dh-jac.net/db/maps/search_portal.php)

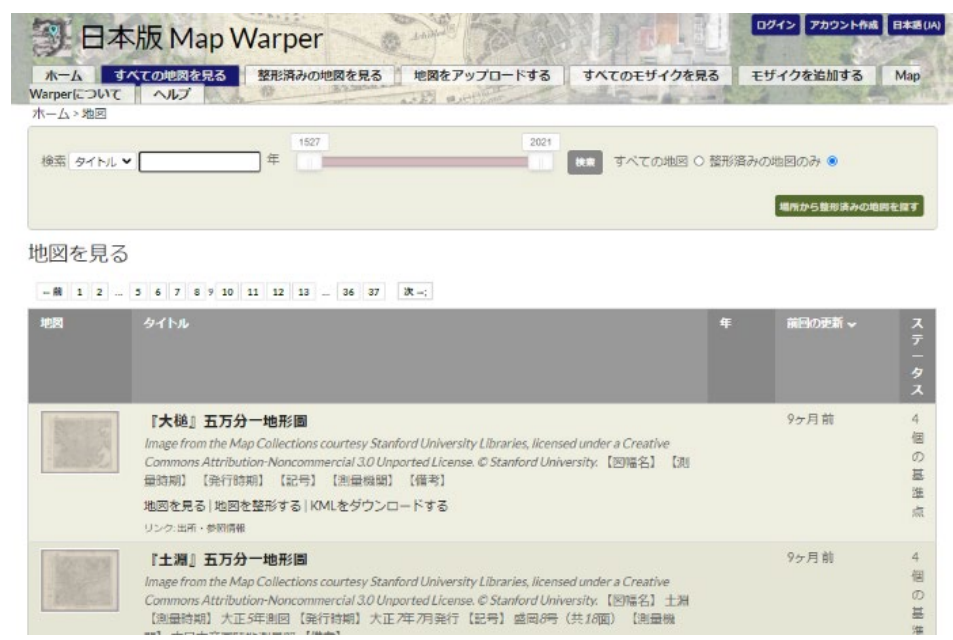

Figure 2. The Japanese version of Map Warper.

(https://mapwarper.h-gis.jp/)

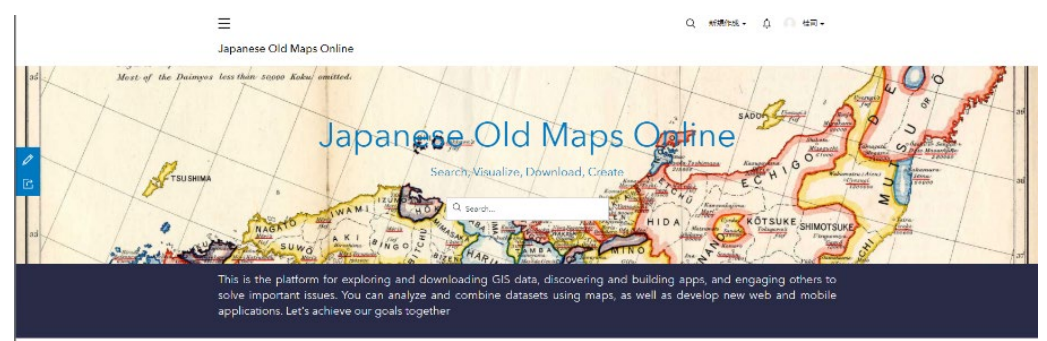

$$
\text { Explore your data }
$$

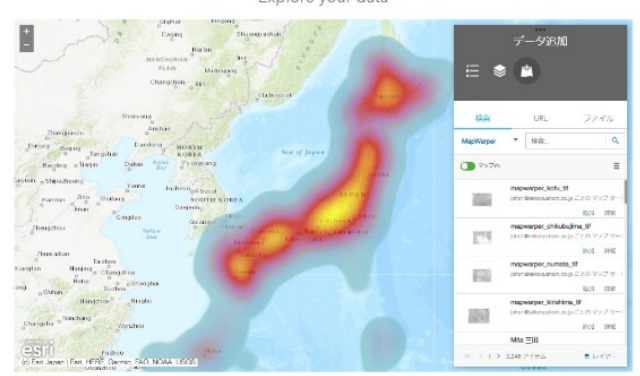

Figure 3. Japanese Old Maps Online.

(https://japanese-old-maps-online-rstgis.hub.arcgis.com/) 